\title{
REVIEW
}

\section{Polychlorinated biphenyls (PCBs) and neurological development in children: a systematic review}

\author{
N Ribas-Fitó, M Sala, M Kogevinas, J Sunyer
}

\begin{abstract}
Background-Polychlorinated biphenyls (PCBs) are complex mixtures of persistent contaminants that are widespread in the environment. Newborns are exposed across the placenta and through breast feeding. Experimental animal studies have indicated that PCBs are neurotoxic. The neurological effects of these compounds on children are not clear. Methods-A systematic review of literature on the relation between neurological development in children and exposure to polychlorinated biphenyls.

Results-Seven follow up studies evaluated the effect of prenatal exposure to PCBs. Two of these studies evaluated highly exposed children. In newborns, an increase of the abnormal reflexes was observed in all four studies evaluating it. During the first months of life, a decrease in motor skills was observed in four of the five studies that investigated psychomotor development; deficits in the acquisition of cognitive skills were observed only in one study assessing non-highly exposed populations. At 4 years of age, an effect on the cognitive areas was observed in four of the five studies that evaluated it. Postnatal exposure to PCBs through breast feeding was not clearly related to any effect on neurological development.

Conclusions-These studies suggest a subtle adverse effect of prenatal PCBs exposure on child neurodevelopment. Differences in study design, inconsistency in some of the results, and the lack of adequate quantitative exposure data, do not allow the derivation of the degree of risk associated with neurodevelopmental effects at current levels of exposure. (F Epidemiol Community Health 2001;55:537-546)
\end{abstract}

There is growing evidence that some environmental chemicals can interrupt neurodevelopmental processes during critical periods of development, resulting in effects on behaviour, or cognitive function. ${ }^{1}$ Awareness of the toxicity of organochlorine compounds was first established after two well documented episodes of accidental exposure to polychlorinated biphenyls (PCBs) via contaminated cooking oil in
Japan (Yusho) ${ }^{2}$ and Taiwan (Yucheng). ${ }^{3}$ Public health interest about the potential impact of environmental exposure to organochlorine compounds on child neurodevelopment has recently increased and several observational studies have been conducted. ${ }^{4-32}$ However, the considerable heterogeneity among these studies in the selection of adequate markers of exposure, in the measure of the outcomes and in the control for possible confounder factors complicates the derivation of firm conclusions about the possible neurodevelopmental effects of these compounds. $^{33} 34$

\section{Properties of PCBs}

PCBs are complex mixtures of persistent contaminants that are ubiquitous in the environment. They have been in widespread use, since the 1930s, as dielectric fluids in transformers and capacitors and in a variety of other applications. ${ }^{35}$ Most, but not all, ${ }^{36}$ industrialised countries have now banned or severely restricted their production.

PCBs are a family of chemicals including 209 different congeners. However, the number of the congeners present in extracts from biotic samples is much lower than the theoretical numbers. ${ }^{37}$ The mechanisms of toxicity of these congeners are not similar and the doses at which they are active vary by orders of magnitude. ${ }^{38}$ The planarity of the molecule is very important in both biostability and toxicology. Planar PCBs bind to the aryl-hydrocarbon (Ah) receptor resulting in some dioxin-like effects. ${ }^{39}$

Because of their high biostability and lipophilicity and because they are resistant to both chemical and biological degradation they accumulate in food chains and can be found in the tissues of wildlife, domestic animals, and humans worldwide. ${ }^{40}$ Nowadays the sources of human exposure to PCBs are food, particularly fish and fish products and animal fats. PCBs are preferentially stored in adipose tissue and are also present, to a smaller extent, in serum and human milk. The concentrations of PCBs in the different organs depend on the lipid content of such organs, with the exception of the brain, where the concentration is lower than the lipid content would indicate. These lower concentrations are attributable to the nature of brain lipids that are more polar than 
adipose tissue lipids. ${ }^{41}$ PCBs also pass through the placenta and through human milk.

\section{Experimental studies on neurotoxicity of PCBs}

During the past 20 years, there has been an attempt to understand the cellular bases of PCB induced behavioural and neurological effects in animal and in vitro models. In vitro models have shown a decrease of cell dopamine content after PCB exposure. ${ }^{42-44}$ Experimental studies with laboratory rodents and non-human primates have indicated changes in dopamine function. ${ }^{45-47}$ Changes in locomotion activity, ${ }^{48} 49$ in behaviour ${ }^{5051}$ and in learning and memory functions in the adult animal ${ }^{485}$ have also been described after prenatal exposure to PCBs. These effects have been mainly described for non-dioxin-like ortho substituted PCBs congeners $^{53}$ as well as for the lower chlorinated PCBs. ${ }^{54}$ Recent research also suggests that PCBs can change a number of other physiological processes that may be important for development. For example, PCB induced alteration in thyroid function during development may underline some of the developmental effects of PCBs reported in humans and animal models..$^{556}$

\section{Assessment of neurodevelopment in newborns and infants}

Interpretation of studies on possible adverse developmental effects in infants from perinatal exposure to PCBs, in contrast with experimental models, is hampered by a high number of potential confounding factors and by difficulties in evaluating exposure and outcome measures. In studying human development, besides behaviour and cognition, it is difficult to define what is "normal". Human behaviour and intellect has been assessed by comparison to the "norm", and for this reason, different developmental tests based on general population development have been created. Neonatal tests mainly assess tonicity, reflexes, alertness, responsiveness and state regulation of the newborn. Child tests are constructed with the premise that an ability may or may not have been acquired in a given age for the children. ${ }^{57}$
Child tests are divided into mental (that is, language, cognition, memory and social patterns), motor (that is, gross and fine motor functions) and behavioural (that is, activity rating and behaviour) scales. These tests are reliable, well standardised and have specific items for the assessment of motor, mental and behavioural development. Their use facilitates comparison of results from the different studies. All mental and motor standardised tests have a mean full scale score around 100, with the exception of the Fagan Test of Visual Recognition Memory (FTVRM), which calculates the percentage of success. For the behavioural tests a higher score indicates a higher frequency of behavioural or activity problems in the child. The neurodevelopmental assessment tools mentioned in this review are briefly described in table 1 .

\section{Epidemiological studies}

IDENTIFICATION OF EPIDEMIOLOGICAL STUDIES AND ANALYSIS

All the available studies about the effects of PCBs on children's neurobehavioural development published from 1976 to January 2000 were reviewed. We searched the computerised database of Medline by using "polychlorinated biphenyls", "development", and "neurotoxicology". We also reviewed conference proceedings of related topics as well as citations in the identified reports. We only included papers that had been written in English. A total of 29 publications coming from seven independent populations were identified.

Results have been grouped by effect studied (motor, mental or behavioural) and age at evaluation (newborn ( $<1$ month) and children (>3 months-11 years)). Some studies presented the mean scores of the test used, while others provided a $\beta$ coefficient of the increment or decrement in the scale score as a function of PCB exposure. To allow comparisons among all studies, we presented $95 \%$ confidence intervals of the mean difference or the $\beta$ coefficients, which were derived from standard deviations or from graphical presentations. ${ }^{8911}$ As in one publication $^{32}$ the scores were reported in a raw form, we transformed them to the standard index score using the Bayley scales for infant development (2nd ed)..$^{57}$

Table 1 Standardised neurodevelopmental tests mentioned in the publications reviewed

\begin{tabular}{|c|c|c|c|}
\hline Test & Age & Assessment & Reference \\
\hline \multicolumn{4}{|l|}{ Neonatal tests } \\
\hline Brazelton neonatal scales (BNBAS) & $<3$ days & Reflexes, responsiveness, state regulation & 67 \\
\hline Newborn neurological exam (Prechtl) & $10-21$ days & Age-appropriated neurodevelopment & 24 \\
\hline \multicolumn{4}{|l|}{ Children tests } \\
\hline \multicolumn{4}{|l|}{ Mental } \\
\hline Fagan test of visual recognition memory (FTVRM) & 3-12 months & Novelty preference; short-term memory; "infant intelligence" & 80 \\
\hline Bayley scales of infant development (MDI) & $<2.5$ years & Age-appropriate cognitive ability & 57 \\
\hline Stanford-Binet (SB) & $2.5-6$ years & General intelligence & 66 \\
\hline Kaufman assessment battery for children (KABC) & $2.5-12.5$ years & Age-appropriate cognitive ability & 66 \\
\hline McCarthy scales of children's abilities (MCSCA) & $>3$ years & Age-appropriate cognitive ability & 66 \\
\hline Wechsler Intelligence Scale for children (WISC) & $>6$ years & General intelligence & 66 \\
\hline Reynell developmental scales (RDS) & - & Age-appropriate development of language & 28 \\
\hline Chinese Child Developmental Inventory (CCDI) ${ }^{\star}$ & $6 \mathrm{~m}-6 \mathrm{y}$ & General developmental status $\dagger$ & 12 \\
\hline \multicolumn{4}{|l|}{ Motor } \\
\hline The Neurological exam for toddler age (Hempel) & Toddlers & Age-appropriate motor ability & 27 \\
\hline Bayley scales of infant development (PDI) & $<2.5$ years & Age-appropriate motor ability & 57 \\
\hline \multicolumn{4}{|l|}{ Behavioural } \\
\hline Rutter's child behaviour scale (RCBS-A) & $0-12$ years & Child behavioural problems, hyperactivity & 66 \\
\hline Werry-Weiss-Peters activity scale (WWPAS) & $3-11$ years & Children's activity level & 66 \\
\hline
\end{tabular}

*Adopted and modified in 1978 from Minnesota Child Developmental Inventory. †CCDI has seven subscales: two for motor development and five for mental development. 


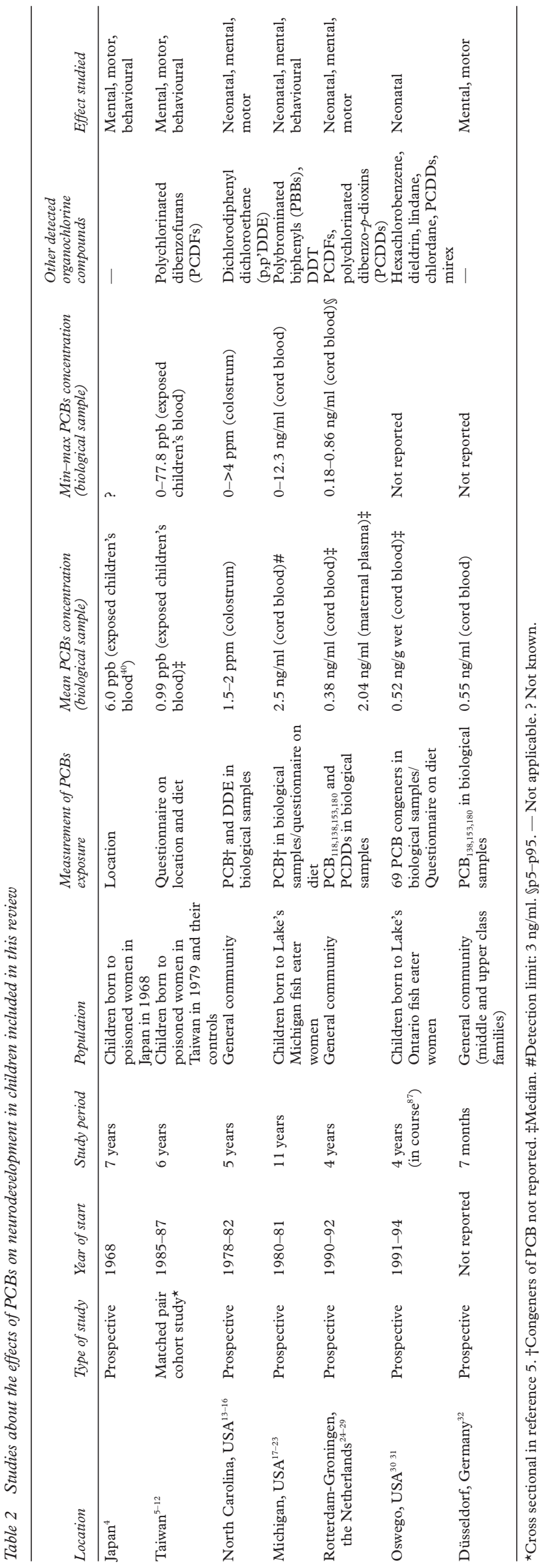

CHARACTERISTICS OF THE STUDIES

Table 2 summarises the design and reporting of the seven studies considered in this review. All studies were prospective. The Japanese and the Taiwanese studies reported data from children highly exposed to PCBs. In Taiwan, for every exposed child, two control children matched on neighbourhood, age, sex, mother's age and parent's combined education level were used. The studies in Michigan and Oswego were based on children born to women who ate contaminated fish. The other three studies were based on children from general populations. The measure of exposure varied significantly between studies. In the Japanese and Taiwanese studies, information of children's exposure was only based on place of residence and on a mother's questionnaire on diet, respectively. However, in both studies, levels of PCBs in exposed children's sera were analysed in a subsample. In the rest of the studies information of children's exposure was specifically based on levels of PCBs measured in different biological samples. In Michigan and in Oswego, a questionnaire was also used to assess exposure to PCBs. Prenatal exposure was estimated through the measure of PCBs in cord blood and maternal plasma close to delivery or colostrum. Postnatal exposure was measured through the quantification of PCBs in milk samples or in child sera. Specific information about the congeners analysed was only given in the Netherlands, German and Oswego studies. The North Carolina and the Netherlands cohorts also studied the effects of other organochlorine compounds on neurodevelopment in children. The reported PCBs concentrations among children (sera or cord blood) in the highly exposed cohorts (Japan, Taiwan) seem to be orders of magnitude higher than among children from general populations (the Netherlands and Germany). Levels of PCBs in the sera of Yusho children are the highest.

NEURODEVELOPMENTAL EFFECTS OF PCB

EXPOSURE AMONG NEWBORNS

A neonatal effect was studied in North Carolina, Michigan, the Netherlands and Oswego. Results from all the four studies suggest some negative effects on neonatal development. In North Carolina, higher PCB levels in maternal milk at birth were associated with hypotonicity and hyporeflexia. ${ }^{13}$ Maternal consumption of contaminated fish in Michigan was predictive of newborn motor immaturity, poorer lability of states, a greater amount of startle, and more abnormally hypoactive reflexes in the Neonatal Behavioral Assessment Scale (NBAS) ${ }^{17}$ but higher cord serum PCB levels were not related to any adverse outcomes. In the Netherlands, exposure through breast milk to PCBs, polychlorodibenzo-p-dioxins (PCDDs) and polychlorodibenzofurans (PCDFs) was associated with a reduced neonatal neurological optimality, but levels of PCB in cord blood were not related to nervous system dysfunction. ${ }^{24}$ The Oswego study found that newborns highly exposed to highly chlorinated PCBs scored more poorly on the autonomic and habituation 


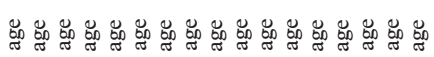

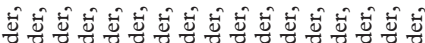

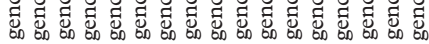

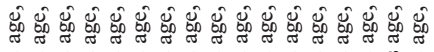
के a

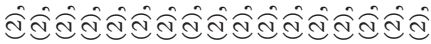

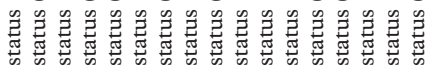
草.

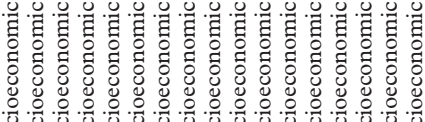

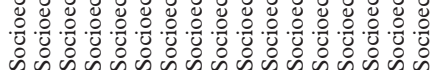

क

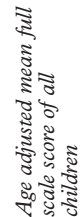

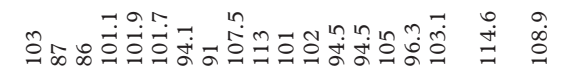

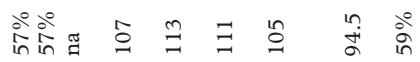

竞

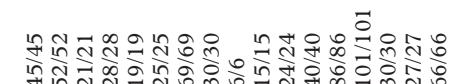

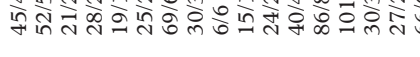

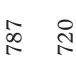

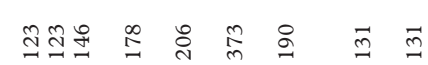

竎

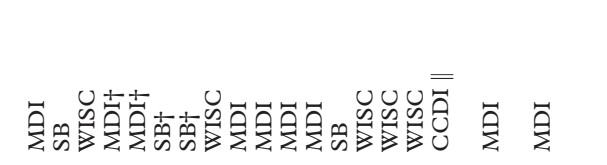

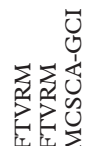

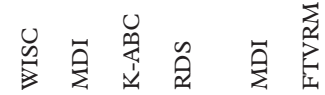

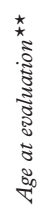

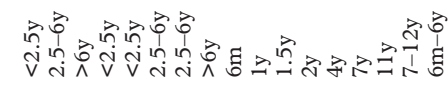

$\geq$

舁

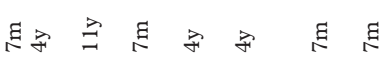

डิ

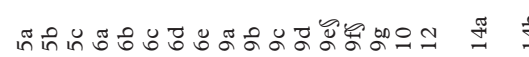

\&

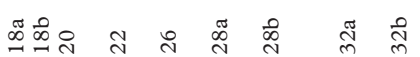
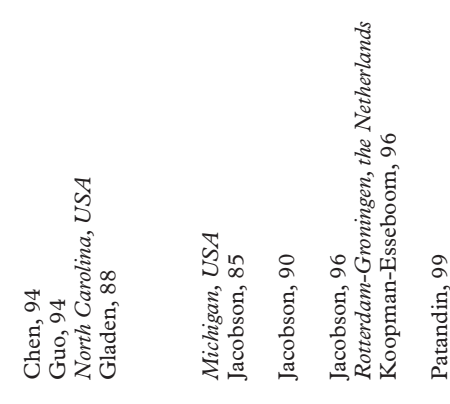

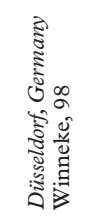

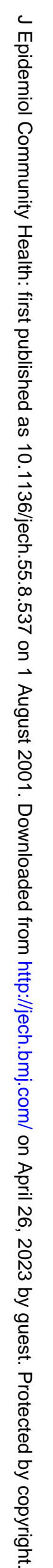




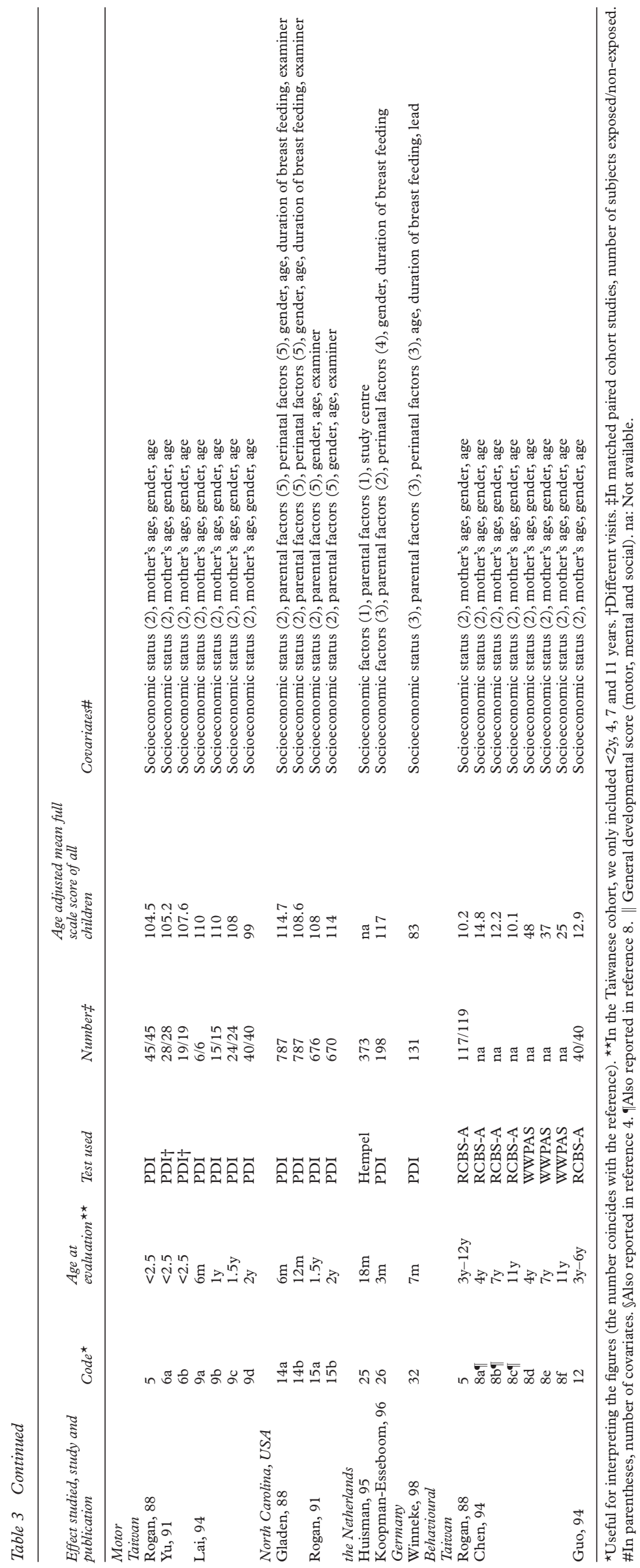

\begin{abstract}
KEY POINTS
- Health risk of the human intake of persistent organochlorine compounds to child neurodevelopment is a matter of worldwide concern.

- The existing research suggests that polychlorinated biphenyls (PCBs) hinder neurodevelopment to children exposed early in life.

- Considerable heterogeneity between study designs does not permit a joint quantitative measure of the association.

- The universal exposure to organochlorine compounds makes necessary further research of populations exposed to the current levels of these contaminants.
\end{abstract}

clusters of the NBAS and, although not significantly, had more abnormal reflexes. ${ }^{30} 31$

NEURODEVELOPMENTAL EFFECTS OF PCB

EXPOSURE AMONG CHILDREN

Prenatal exposure to PCBs

Original quantitative data of the effects of prenatal exposure to PCBs on children from 6 months to 11 years were reported in 15 publications from Taiwan, North Carolina, Michigan, the Netherlands and Germany (table 3).

A negative association between PCBs and mental development was found in children born to mothers who had consumed contaminated oil in 1979 in Taiwan (fig 1A). In most of the evaluations at different ages, the average difference between exposed and non-exposed was around -4 to -6 points. In the studies based on non-highly exposed populations a negative effect on the acquisition of cognitive skills during the first months of life was only observed in the Michigan study (fig 1B). In addition, a significant association between prenatal PCBs exposure and mental impairment was observed at 4 years of age in the Netherlands and 11 years in Michigan (fig 1B). When memory was specifically assessed, an inverse association could also be observed in Michigan at 4 years of age..$^{20}{ }^{21}$ In contrast, the North Carolina study reported that no effects were observed at 3,4 or 5 years. ${ }^{16}$

An inverse association with the psychomotor scale scores was also observed both in children prenatally exposed in Taiwan and in children highly exposed in North Carolina compared with the non-exposed and the less exposed, respectively (fig 2A). In North Carolina and in the Netherlands, negative associations with psychomotor scales were observed during the first year of life, particularly at 3 months (fig 2B). In the Netherlands study the authors also reported that cord blood PCB levels did not predict poorer scores in motor development at either 7 months ${ }^{26}$ or at 42 months. ${ }^{27}$

Behavioural scales were only studied in Taiwan and Michigan. In all the publications from the Taiwanese study there was a negative association between children born to women who had consumed contaminated oil and higher scores in the behavioural scales (fig 3). In Michigan, prenatal PCB exposure was not 

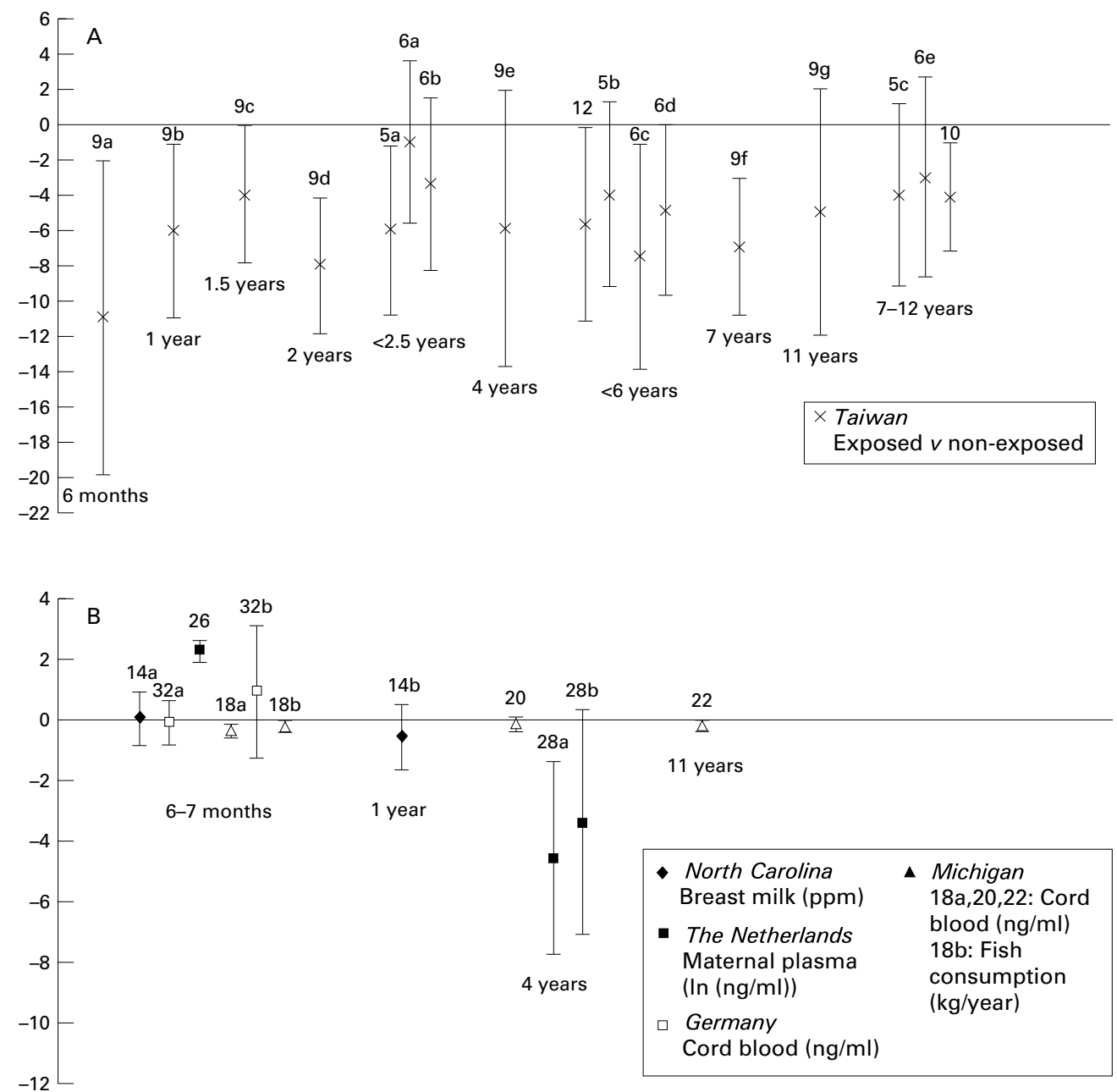

Figure 1 Mean difference in full mental scales (and 95\% confidence intervals): (A) between prenatally exposed and non-exposed children to PCBs and $(B)$ for a change in one unit of prenatal exposure to PCBs; ordered by age of the infant and type of test of the different studies.

associated with worse behavioural score in either the composite activity rating ${ }^{19}$ or sustained attention rating ${ }^{21}$ at 4 years of age.

The Japanese study did not use any specific standardised test and did not report quantitative data. However, a clinical examination of 127 exposed children showed that they had a mean IQ of 70, had hypotonia and appeared to be sullen, expressionless and hypoactive. ${ }^{4}$ Overall, in addition to the effects found in the Japanese and the Taiwanese accidents, prenatal PCB exposure seems to be related to a decrease in motor skills during the first months of life and to an effect on the cognitive areas among children older than 4 years.

POSTNATAL EXPOSURE TO PCBS

Postnatal exposure was studied in North Carolina, Michigan, the Netherlands and Germany. Unlike prenatal exposures, postnatal exposures have rarely been found to be associated with any neurodevelopmental effect. In North Carolina, postnatal exposure to PCBs, based on a function of the concentration of the chemical in milk and the duration of breast feeding, was not related to worse performance in any of the tests and ages studied. ${ }^{14-16}$ In Michigan, levels of PCBs in maternal milk were unrelated to recognition memory performance at 7 months, ${ }^{18}$ cognitive performance at 4 years, ${ }^{20}{ }^{21}$ and at 11 years. ${ }^{22}$ Only the composite activity rating at 4 years was negatively related to both the maternal milk PCBs level and the four year serum PCBs level. ${ }^{19}$ In the Netherlands, postnatal exposure to PCBs was analysed together with the effects of dioxins. According to the toxic equivalent factor of each congener, a total of 17 dioxins and three PCBs were added and summarised as a total PCBdioxin toxic equivalent concept (TEQ). Examination of the postnatal exposure revealed no significant effect of the total PCB-dioxin TEQ exposure at 3 months of age. At 7 months higher amounts of PCBs and dioxin exposure through breastfeeding had a significant adverse effect on the psychomotor outcome among breast feeders. ${ }^{26}$ At 18 months ${ }^{25}{ }^{26}$ and at 24 months, ${ }^{27}{ }^{28}$ an effect of lactational exposure to these compounds could not be detected. ${ }^{25}{ }^{26}$ In Germany, only the Bayley mental developmental index exhibited a significant negative association with PCBs levels in breast milk at 7 months. ${ }^{32}$

\section{Discussion}

The reviewed studies have shown that PCBs exposure through the mother might be related 


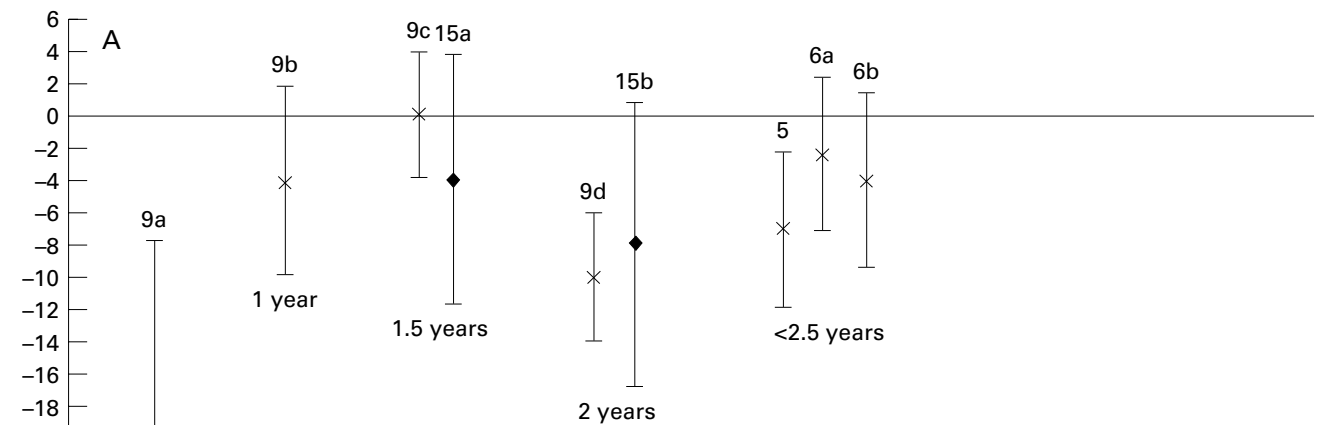

\begin{tabular}{|l|}
$\times$ Taiwan \\
Exposed $v$ non-exposed \\
- North Carolina \\
$>4 p p m v<0.09$ \\
ppm milk
\end{tabular}
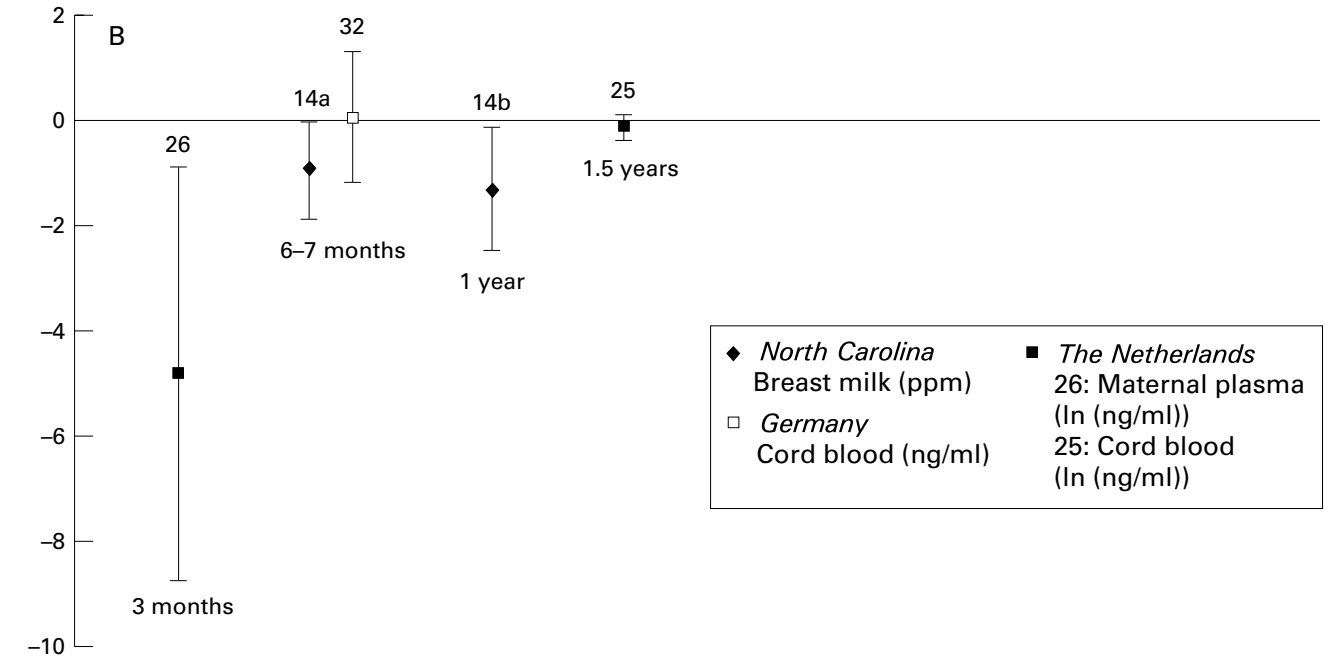

Figure 2 Mean difference in full motor scales (and 95\% confidence intervals): (A) between prenatally exposed and non-exposed children to PCBs and $(B)$ for a change in one unit of prenatal exposure to PCBs; ordered by age of the infant and type of test of the different studies.

to some small adverse effects on the neurodevelopment and behaviour of children. Seven follow up studies evaluated the effect of prenatal exposure to PCBs. Two of these studies evaluated highly exposed children. In newborns, an increase of the abnormal reflexes was observed in all four studies evaluating it. During the first months of life, a decrease on the motor skills was observed in four of the five studies that investigated psychomotor development; deficits in the acquisition of cognitive skills were observed only in one study assessing non-highly exposed populations. The studies based on general populations mainly evaluated motor development in infants up to 2 years, so it cannot be excluded that the observed psychomotor effects during the neonatal period and early infancy might disappear later in life. At 4 years of age, an effect on the cognitive areas was observed in four of the five studies that evaluated it. The strongest effects on motor and mental development were observed in Taiwan, in the reports at the age of 6 months. A possible explanation could be related to the fact that exposed mothers were encouraged not to breast feed their infants, ${ }^{58}$ impeding the possible neurodevelopmental benefits of breast feeding to their newborn. ${ }^{59}{ }^{60}$ Effects on neurological development of postnatal exposure to PCBs through breast feeding were inconsistent among the studies and preponderantly nonsignificant. PCBs exposure is higher through breast feeding than in utero, ${ }^{58}{ }^{61}$ but intrauterine exposure seems to pose a greater threat to the infant than postnatal exposure as has been observed for other chemicals. ${ }^{62}$

Exposure to PCBs was assessed through biological samples or questionnaires on residence or diet. Questionnaires did not specifically discriminate between prenatal or postnatal exposure and were not sufficiently sensitive to detect either exposure to specific compounds or the magnitude of the exposure. In Taiwan, the authors tried to improve the exposure measurement provided by the questionnaire by using the physical signs observed in the children after PCBs intoxication, such as presence of nail abnormalities. No relation 


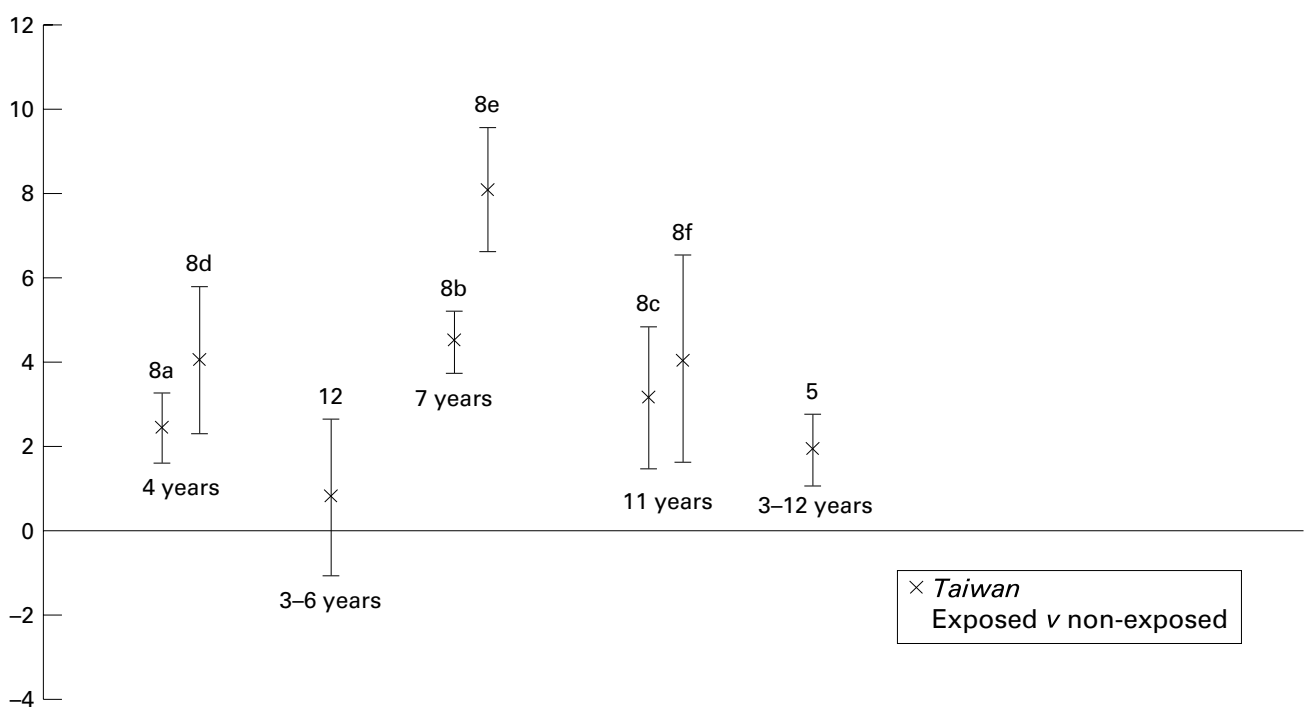

Figure 3 Mean difference in full behavioural scales (and 95\% confidence intervals) between prenatally exposed and non-exposed children to PCBs; ordered by age of the infant and type of test of the different studies.

between severity of the external clinical examination and the neurodevelopmental effects could be observed. ${ }^{6}$ However, the measurement of exposure through an index of physical signs could be less valid than the questionnaire as individual susceptibility was not taken into account. Problems with biological measures of exposure were of a different nature. Some studies measured prenatal exposure of newborns through assays in maternal blood or milk close to delivery rather than cord blood. Although there is a correlation between maternal and child levels, ${ }^{63}$ a within individual variability can be observed in the correlation between levels of specific PCBs' congeners in maternal milk close to delivery and levels in cord blood. ${ }^{64}$ Differences in the analytical methods used could also explain some of the differences observed among studies. In Michigan and in North Carolina exposure was defined from total PCBs levels by summing different Webb-McCall peaks. ${ }^{65}$ This method may have hampered the identification of specific PCB congeners, such as the non-dioxin like PCBs or the less chlorinated, which have been described to be the most neurotoxic in animals. $^{54}$

The measure of the outcome was also significantly different among the studies. The age at evaluation and the specific use of the available standardised tests differed in each report. Mental development was assessed in all studies at 6-7 months of age. Only some of these studies followed up their children up to school ages. Motor development was mainly evaluated in children aged less than 2 years. The behavioural and mental repertoires of the younger child are more vulnerable to acute external influences difficult to control for than the motor skills, and therefore less reliable. ${ }^{66}$ Clusters like mobility or reflexes, which predict better future motor function, tend to respond less to the environmental stimuli during the examination. ${ }^{67}$ In addition, the mental skills early in life are less predictive of later functioning than motor skills. ${ }^{68}$
The major differences between studies laid in the methods of controlling for confounders. Socioeconomic status, prenatal maternal alcohol consumption, maternal smoking during pregnancy, maternal age, dietary habits, gender, the caregiving environment as measured by the HOME inventory ${ }^{69}$ and breast feeding itself are strongly associated with neurodevelopment, ${ }^{70}$ and adjustment for these factors was heterogeneous among studies and not comparable. Besides, humans are exposed to PCBs that are quite frequently present in mixtures with other organochlorine compounds or other chemical products, but only a few of the studies measured other compounds. Other organochlorine compounds were studied in North Carolina, Michigan and Oswego. The studies in Michigan, Germany and Oswego took into account levels of lead. Only the study in Oswego controlled for methylmercury, which has been reported to act synergistically with polychlorinated biphenyls in in vitro models, ${ }^{71}$ and has been shown to be associated with deficits in language, attention and memory in children. ${ }^{72}$ In a recent study in the Faroe Islands, an association with PCBs exposure disappeared after adjustment for methyl mercury. ${ }^{73}$

Systematic reviews are prone to publication bias $^{74}$ as those studies with negative findings are less likely to be published. However, it seems unlikely that any large study might have not been published because of negative findings. We are quite confident that all published studies about the effects of PCBs on child neurodevelopment have been considered in this review, but publication bias cannot be absolutely discarded.

Despite the relatively consistent results due to prenatal exposures, the lack of homogeneity between study designs does not allow having a joint quantitative measure of the association. The present studies do not provide enough information on type of dose-response relation and the presence of a threshold level. Taiwan and Japan, which had the highest levels of PCBs and PCDFs, did not use levels of 
internal dose of PCBs to assess the association with neurodevelopmental tests and only quantified exposure in two levels (exposed/nonexposed). The critical temporal period during the brain growth spurt where the potential damage might occur is also not known. Measurements at birth averaged all prenatal exposures and could not differentiate acute exposures during pregnancy. Moreover, information on the possible transience of the effects, or the specific cognitive skills that might remain permanently affected in later childhood cannot be derived from the present studies. As was fully assessed in the case of lead, it is important to clarify the possible reversibility of the observed effects. ${ }^{75}$

Another important issue of concern is whether the small group average effects observed have a clinical significance at the individual level. ${ }^{76}$ At current levels in the Great Lakes region, the effects of intrauterine PCBs exposure appeared to be so subtle that they would not have been evident in a routine clinical examination. ${ }^{77}$ Studies on lead exposure have shown that a decrease of 4 points in average population intelligence increases the number of children with IQs of $<80$ by threefold and decreases the percentage of children with IQ scores of $>125 .^{78}$ In a similar way, exposure to these ubiquitous environmental pollutants may have a seemingly small effect at individual level, but probably have a large impact at population level. ${ }^{79}$

We conclude that the available studies suggest an adverse effect of prenatal PCBs exposure on neurological development in children. However, differences in study design, some inconsistencies in the results, and the lack of homogeneous quantitative exposure data, do not allow the derivation of the degree of risk associated with neurodevelopmental effects at current levels of exposure. Future investigation, as well as ongoing cohort studies, ${ }^{80-84}$ should guarantee standard evaluation of specific organochlorine exposure using biological samples, homogeneous outcome assessment through standardised tests and measurement of important covariates such as socioeconomic factors and other neurotoxic chemicals. ${ }^{85}$ The universal exposure to these compounds and the importance of early assessment of signs of dysfunction to provide the best possible basis for decisions on prevention, ${ }^{86}$ makes necessary the setting up and continuation of large follow up studies of populations exposed to the current levels of these contaminants.

We are grateful to Dave Macfarlane for his help in editing this manuscript.

Funding: supported in part by grants from "Fundació La Caixa" 97/009.00, and Generalitat de Catalunya-CIRIT 1999SGR 00241

Conflicts of interest: none.

1 Spyker JM. Assessing the impact of low level chemicals on development: behavioral and latent effects. Fed Proc 2000;34:1835-44

2 Kuratsune M, Yoshimura T, Matsukaza J, et al. Yusho, a poisoning caused by rice oil contaminated with polychlorinated biphenyls. HSMHA Health Rep 1971;86:1083-91.

3 Hsu ST, Ma CI, Hsu KH, et al. Discovery and epidemiology of PCB poisoning in Taiwan: a four-year follow-up. Environ of PCB poisoning in Taiwan: a
4 Harada M. Intrauterine poisoning: clinical and epidemioogical studies and significance on the problem. Bull Inst Const Med Kumamoto Univ 1976;25:1-69.

5 Rogan WJ, Gladen BC, Hung KL, et al. Congenital poisoning by polychlorinated biphenyls and their contaminants in Taiwan. Science 1988;241:334-6.

6 Yu ML, Hsu CC, Gladen BC, et al. In utero PCB/PCDF exposure: relation of developmental delay to dysmorphology and dose. Neurotoxicol Teratol 1991;13:195-202.

7 Chen YCJ, Guo YL, Hsu CC, et al. Cognitive development of Yucheng ('oil disease') children prenatally exposed to heat-degraded PCBs. $¥ A M A$ 1992;268:3213-18.

8 Chen YCJ, Yu ML, Rogan WJ, et al. A 6-year follow up of behavior and activity disorders in the Taiwan Yucheng chilbehavior and activity disorders in the Taiw
dren. Am $\mathcal{f}$ Public Health 1994;84:415-21.

9 Lai TJ, Guo YL, Yu ML, et al. Cognitive development in Yucheng children. Chemosphere 1994;29:2405-11.

10 Chen YJ, Hsu CC. Effects of prenatal exposure to PCBs on the neurological function of children: a neuropsychological and neurophysiological study. Dev Med Child Neurol 1994; 36:312-20.

11 Yu ML, Hsu CC, Guo YL, et al. Disordered behavior in the early-born Taiwan Yucheng children. Chemosphere 1994; 29:2413-22.

12 Guo YL, Chen YC, Yu ML, et al. Early development of Yucheng children born seven to twelve years after the TaiYucheng children born seven to twelve years after the

13 Rogan WJ, Gladen BC, McKinney JD, et al. Neonatal effects of trasnplancental exposure to PCBs and DDE. 7 Pediatr 986;109:335-41.

14 Gladen BC, Rogan WJ, Hardy P, et al. Development after exposure to polychlorinated biphenyls and dichlorodiphenyl dichloroethene transplacentally and through human milk. F Pediatr 1988;113:991-5.

15 Rogan WJ, Gladen BC. PCBs, DDE, and child development at 18 and 24 months. Ann Epidemiol 1991;1:407-13.

16 Gladen BC, Rogan WJ. Effects of perinatal polychlorinated biphenyls and dichlorodiphenyl dichloroethene on later development. F Pediatr 1991;119:58-63.

17 Jacobson JL, Jacobson SW, Schwartz PM, et al. Prenatal exposure to an environmental toxin: a test of the multiple exposure to an environmental toxin: a test of

18 Jacobson SW, Fein GG, Jacobson JL, et al. The effect of intrauterine PCB exposure on visual recognition memory. Child Dev 1985;56:853-60.

19 Jacobson JL, Jacobson SW, Humphrey HE. Effects of exposure to PCBs and related compounds on growth and activity in children. Neurotoxicol Teratol 1990;12:319-26.

20 Jacobson JL, Jacobson SW, Humphrey HEB. Effects of in utero exposure to polychlorinated biphenyls and related contaminants on cognitive functioning in young children. $\mathcal{F}$ Pediatr 1990;116:38-45.

21 Jacobson JL, Jacobson SW, Padgett RJ, et al. Effects of prenatal PCB exposure on cognitive processing efficiency and natal PCB exposure on cognitive processing efficie
sustained attention. Dev Psychol 1992;28:297-306.

22 Jacobson JL, Jacobson SW. Intellectual impairment in children exposed to polychlorinated biphenyls in utero. $N$ Engl f Med 1996;335:783-9.

23 Jacobson SW, Jacobson JL, Fein GG. Environmental toxins and infant development. In: Fitzgerald HE, Lester BM, Yogman MW, eds. Theory and research in behavioral pediatrics. Vol 3. New York, NY: Plenum Press, 1986:96-146.

24 Huisman M, Koopman-Esseboom C, Fidler V, et al. Perinatal exposure to polychlorinated biphenyls and dioxins and its effect on neonatal neurological development. Early Hum Dev 1995;41:111-27.

25 Huisman M, Koopman-Esseboom C, Lanting CI, et al. Neurological condition in 18-month-old children perinatally exposed to polychlorinated biphenyls and dioxins. Early Hum Dev 1995;43:165-76.

26 Koopman-Esseboom C, Weisglas-Kuperus N, Ridder MAJ, et al. Effects of polychlorinated biphenyl/dioxin exposure and feeding type on infants' mental and psychomotor development. Pediatrics 1996;97:700-6.

27 Lanting CI, Patandin S, Fidler V, et al. Neurological condition in 42-month-old children in relation to pre- and postnatal exposure to polychlorinated biphenyls and dioxins. Early Hum Dev 1998;50:283-92.

28 Patandin S, Lanting CI, Mulder PGH, et al. Effects of environmental exposure to polychlorinated biphenyls and dioxins on cognitive abilities in Dutch children at 42 months of age. F Pediatr 1999;134:33-41.

29 Vreugdenhil H, Lanting C, Patandin S, et al. Prenatal and postnatal PCB and dioxin exposure and cognitive abilities in the Dutch cohort at school age. Dioxin 99, 19th International Symposium on Halogenated Environmental International Symposium on Halogenated Environmental Organic Pollutants and POP.

30 Lonky E, Reihman J, Darvill T, et al. Neonatal Behavioral Assessment Scale performance in humans influenced by maternal consumption of environmentally contaminated lake Ontario fish. $\mathcal{F}$ Great Lakes Res 1996;22:198-212.

31 Stewart P, Reihman J, Lonky E, et al. Prenatal PCB exposure and neonatal behavioral assessment scale

Winneke G, Bucholski A, Heinzow B, et al. Developmental neurotoxicity of polychlorinated biphenyls (PCBs): cognitive and psychomotor functions in 7-month old children. Toxicol Lett 1998;102-103:423-8.

33 Jacobson JL, Jacobson SW. Sources and implications of interstudy and interindividual variability in the developmental neurotoxicity of PCBs. Neurotoxicol Teratol 1996;18: 257-64. 
34 Seegal RF. Can epidemiological studies discern subtle neurological effects due to perinatal exposure to PCBs? Neurotoxicol Teratol 1996;18:251-4.

35 International Programme on Chemical Safety. Polychlorinated biphenyls and terphenyls. 2nd ed. Geneva: World Health Organization, 1993

36 Carpenter DO. Polychlorinated biphenyls and human health. Int f Occup Med Environ Health 1998;11:291-303.

37 Brouwer A, Ahlborg U, Van Leeuwen FXR, et al. Report of the WHO working group on the assessment of health risks for human infants from exposure to PCDDs, PCDFs and PCBs. Chemosphere 1998;37:1627-43.

38 Giesy JP, Kannan K. Dioxin-like and non-dioxin-like toxic effects of polychlorinated biphenyls (PCBs): implications for risk assessment. Crit Rev Toxicol 1998;28:511-69.

39 Van den BM, Birnbaum L, Bosveld ATC, et al. Toxic equivalency factors (TEFs) for PCBs, PCDDs, PCDFs for humans and wildlife. Environ Health Perspect 1998;106: humans 92 .

40 Jensen AA. Polychlorobiphenyls (PCBs), polychlorodibenzo-p-dioxins (PCDDs) and polychlorodibenzofurans (PCDFs) in human milk, blood and adipose tissue. Sci Total Environ 1987;64:259-93.

41 Dewailly E, Mulvad G, Pedersen HS, et al. Concentration of organochlorines in human brain, liver, and adipose tissue autopsy samples from Greenland. Environ Health Perspect 1999;107:823-8.

42 Chishti MA, Fisher JP, Seegal RF. Aroclors 1254 and 1260 reduce dopamine concentrations in rat striatal slices. $\mathrm{Neu}$ rotoxicology 1996;17:653-60.

43 Shain W, Bush B, Seegal R. Neurotoxicity of polychlorinated biphenyls: structure-activity relationship of individual congeners. Toxicol Appl Pharmacol 1991;111:33-42.

44 Rosin DL, Martin BR. Neurochemical and behavioral effects of polychlorinated biphenyls in mice. Neurotoxicology 1981;2:749-64.

45 Agrawal AK, Tilson HA, Bondy SC. 3,4,3',4'Tetrachlorobiphenyl given to mice prenatally produces lotrachlorobiphenyl given to mice prenatally produces binding sites in caudate nucleus. Toxicol Lett 1981;7:41724.

46 Seegal RF, Pappas BA, Park GA. Neurochemical effects of consumption of Great Lakes salmon by rats. Regul Toxico Pharmacol 1998;27:S68-75

47 Seegal RF, Bush B, Shain W. Lightly chlorinated orthosubstituted PCB congeners decrease dopamine in nonhuman primate brain and in tissue culture. Toxicol Appl Pharmacol 1990;106:136-44

48 Eriksson P. Developmental neurotoxicology in the neonateeffects of pesticides and polychlorinated organic substances. Arch Toxiocol Suppl 1996;18:81-8.

49 Bowman RE, Heironimus MP, Barsotti DA. Locomotor hyperactivity in PCB-exposed rhesus monkeys. Neurotoxicology $1981 \cdot 2 \cdot 251-68$

50 Eriksson P. Developmental neurotoxicity of environmental agents in the neonate. Neurotoxicology 1997;18:719-26.

51 Tilson HA, Kodavanti RS. Neurochemical effects of polychlorinated biphenyls: an overview and identification polychlorinated biphenyls: an overview and identifica

52 Schantz SL, Seo BW, Moshtaghian J, et al. Effects of gestational and lactational exposure to TCDD or coplanar PCBs on spatial learning. Neurotoxicol Teratol 1996;18:30513.

53 Tilson HA, Kodavanti PRS, Mundy WR, et al. Neurotoxicity of environmental chemicals and their mechanism of action. Toxicol Lett 1998;102-103:631-5.

54 Carpenter DO, Arcaro KF, Bush B, et al. Human health and chemical mixtures: an overview. Environ Health Perspect 1998;106:1263-70

55 Osius N, Karmaus W, Kruse H, et al. Exposure to polychlorinated biphenyls and levels of thyroid hormon children. Environ Health Perspect 1999;107:843-9.

56 Tilson HA. Developmental neurotoxicology of endocrine disruptors and pesticides: identification of information gaps and research needs. Environ Health Perspect 1998;106: 807-11.

57 Bayley N. Bayley scales of infant development. 2nd ed. San Antonio: The Psychological Corporation, 1993.

58 Rogan WJ, Gladen BC, McKinney JD, et al. Polychlorinated biphenyls (PCBs) and dichlorodiphenyl dichloroethene biphenyls (PCBs) and dichlorodiphenyl dichloroethene (DDE) in human milk: effects of maternal factors and
vious lactation. Am f Public Health 1986;767:172-7.

59 Boutourline-Young H, Buckley AE, Hamza B, et al. Milk and lactation: some social and developmental correlates among 1000 infants. Pediatrics 1982;69:169-75.

60 Horwood LJ, Fergusson DM. Breastfeeding and later cognitive and academic outcomes. Pediatrics 1998;101:E9.

61 Jacobson JL, Humphrey HEB, Jacobson SW, et al. Determinants of polychlorinated biphenyls (PCBs), polybrominated biphenyls (PBBs), and dichlorodiphenyl trichloroethane (DDT) levels in the sera of young children. $A m \mathcal{F}$ Public Health 1989;79:1401-4.
62 Coyle I, Wayner MJ, Singer G. Behavioral teratogenesis: a critical evaluation. Pharmacol Biochem Behav 1976;4:191200.

63 Jacobson JL, Fein GG, Jacobson SW, et al. The transfer of polychlorinated biphenyls (PCBs) and polybrominated biphenyls (PBBs) across the human placenta and into maternal milk. Am f Public Health 1984;74:378-9.

64 Greizerstein HB, Stinson C, Mendola P, et al. Comparison of PCB congeners and pesticide levels between serum and milk from lactating woman. Environ Res 1999;80:280-6.

65 Jacobson JL, Jacobson SW. Dose-response in perinatal exposure to polychlorinated biphenyls (PCBs): the Michigan and North Carolina cohort studies. Toxicol Ind Health 1996;12:435-45.

66 Spreen O, Strauss E. A Compendium of neuropsychological tests. 1sst edition. New York: Oxford University Press, 1991.

67 Brazelton TB, Nugent JK. Neonatal behavioral assessment scale. 3rd ed. London: Mac Keith Press, 1995.

68 Bornstein MH, Sigman MD. Continuity in mental development from infancy. Child Dev 1986;57:251-74

69 Bradley RH. Children's home environments, health, behavior, and intervention efforts: a review using the HOME inventory as a marker measure. Genet Soc Gen Psychol Monogr 1993; 119:437-90.

70 Jacobson JL, Jacobson SW. Prospective, longitudinal assessment of developmental neurotoxicity. Environ Health Perspect 1996;104:275-83.

71 Bemis JC, Seegal RF. Polychlorinated biphenyls and methylmercury act synergistically to reduce rat brain dopamine content in vitro. Environ Health Perspect 1999; 107:879-85.

72 Grandjean P, Budtz-Jorgensen E, White RF, et al. Methylmercury exposure biomarkers as indicators of neurotoxicity in children aged 7 years. Am 7 Epidemiol 1999;150:301-5.

73 Grandjean P, Weihe P, White RF, et al. Cognitive performance of children prenatally exposed to "safe" levels of methylmercury. Environ Res 1998;77:165-72.

74 Rothman KJ, Greenland S. Modern epidemiology. 2nd ed. Philadelphia: Lippincott, 1998

75 Tong S, Baghurst PA, Sawyer MG, et al. Declining blood lead levels and changes in cognitive function during childhood: the Port Pirie Cohort Study. FAMA 1998;280: 1915-19.

76 Jacobson JL, Jacobson SW. Evidence for PCBs as neurodevelopmental toxicants in humans. Neurotoxicology 1997;18: 415-24.

77 Jacobson JL, Jacobson SW. New methodologies for assessing the effects of prenatal exposure on cognitive functioning in humans. In: Evans MS, ed. Toxic contaminants and ecosystem health: A Great Lakes focus. New York: John Wiley, 1988:373-88.

78 Davis JM. Risk assessment of the developmental neurotoxicity of lead. Neurotoxicology 1990;11:285-91.

79 Schantz SL. Developmental neurotoxicity of PCBs in humans: what do we know and where do we go from here? Neurotoxicol Teratol 1996;18:217-27.

80 Golub M, Jacobson SW. Workshop on perinatal exposure to dioxin-like compounds. IV. Neurobehavioral effects. Environ Health Perspect 1995;103:151-5.

81 Schell LM, Tarbell AM. A partnership study of PCBs and the health of Mohawk youth: lessons from our past and guidelines for our future. Environ Health Perspect 1998;106: 833-40.

82 Hertz-Picciotto I, Charles MJ, Willman E, et al. In utero organochlorine exposures: preliminary results from the child health and development study (CHDS). Epidemiology 1999;10:S99.

83 Schantz SL. Response to commentaries. Neurotoxicol Teratol 1996;18:271-6.

84 Sala M, Ribas-Fitó N, Cardo E, et al. Hexachlorobenzene and other organochlorine compounds incorporation to the new-borns and its effects on neonatal neurological development at 6-8 weeks of life. Dioxin 99, 19th International Symposium on Halogenated Environmental Organic Pollutants and POPs. Venice, Italy: September 1999;44:241-2. [Abstract].

85 Darvill T, Lonky E, Reihman J, et al. Critical issues for research on the neurobehavioral effects of PCBs in humans. Neurotoxicol Teratol 1996;18:265-70.

86 Grandjean P, White RF, Weihe P. Neurobehavioral epidemiology: application in risk assessment. Environ Health Perspect 1996;104:397-400.

87 Daly H, Darvill T, Lonky E, et al. Behavioral effects of prenatal and adult exposure to toxic chemicals found in Lake Ontario fish: two methodological approaches. Toxicol Ind Health 1996;12:419-26. 